\title{
DÉCOMPOSITION ATOMIQUE DES ESPACES DE BERGMAN
}

\author{
F. SYMESAK
}

\begin{abstract}
The aim of this paper is to establish the theorem of atomic decomposition of weighted Bergman spaces $A^{p}(\Omega)$, where $\Omega$ is a domain of finite type in $\mathbb{C}^{2}$. We construct a kernel function $H(z, w)$ which is a reproducing kernel for $A^{p}(\Omega)$ and we prove that the associated integral operator $H$ is bounded in $L^{p}(\Omega)$.
\end{abstract}

\section{Introduction et énoncé des résultats}

Soit $\Omega$ un domaine borné de $\mathbb{C}^{n}$ à bord $\mathcal{C}^{\infty}$ donné par $\Omega=\{z \in$ $\left.\mathbb{C}^{n}, r(z)<0\right\}$. On suppose de plus que $r$ vérifie $|\nabla r(z)|=1$ sur $\partial \Omega=$ $\{z ; r(z)=0\}$. Pour $0<p<+\infty$, on pose $A^{p}(\Omega)=L^{p}(\Omega) \cap \mathcal{H}(\Omega)$, $\mathcal{H}(\Omega)$ désignant l'espace des fonctions holomorphes dans $\Omega$. On note $B$ la projection de Bergman qui est la projection orthogonale de $L^{2}(\Omega)$ sur le sous espace fermé $A^{2}(\Omega)$. La projetée $B f$ de la fonction $f$ de $L^{2}(\Omega)$ est donnée par

$$
B f(z)=\int_{\Omega} B(z, \zeta) f(\zeta) d V(\zeta),
$$

où $B(z, \zeta)$ est le noyau de Bergman et $d V$ la mesure de Lebesgue de $\Omega$.

En 1980, R. Coifman et R. Rochberg ont introduit la notion de décomposition atomique des espaces de Bergman [3]. Dans le cas de la boule unité de $\mathbb{C}^{n}$, ils on montré que toute fonction $f$ de $A^{p}(\Omega)$ peut s'écrire

$$
f(z)=\sum_{i} \lambda_{i} a_{i} B^{q}\left(z, w_{i}\right)
$$

avec $\|f\|_{p} \simeq\left(\sum\left|\lambda_{i}\right|^{p}\right)^{1 / p}$. Dans la relation $1.1, w_{i}$ est une suite de points choisis une fois pour toutes, $q$ est un réel assez grand et $a_{i}$ est une constante de normalisation de sorte que

$$
\left\|a_{i} B^{q}\left(\cdot, w_{i}\right)\right\|_{p}=1
$$


Ainsi $A^{p}(\Omega)$ apparaît comme "engendré" par les $B^{q}\left(\cdot, w_{i}\right)$, mais on ne peut pas parler de base, la décomposition atomique n'étant pas unique.

Cette décomposition a été généralisée par B. Coupet aux domaines strictement pseudo-convexes en remplaçant $B^{q}(z, \zeta)$ par un noyau de Henkin convenable [4].

Notre but est de montrer que les espaces de Bergman possèdent une décomposition atomique lorsque $\Omega \subset \mathbb{C}^{2}$ est un domaine pseudo-convexe de type fini $m \geq 2$. Dans ce cas, le noyau de Bergman a été étudié par A. Nagel, J.-P. Rosay, E. Stein et S. Wainger [9] et par J. Mac-Neal [8]. Il est lié à la géométrie du domaine qui a été décrite par D. Catlin [1]. A tout point $z$ dans un voisinage du bord $U=\left\{z \in \mathbb{C}^{2},|r(z)|<\varepsilon\right\}$, on peut associer un changement de variables $\Phi_{z}$, et la quantité $\tau(z, \delta)$, $\delta>0$, qui traduit l'applatissement du domaine aux points de faible pseudo-convexité. On peut alors considérer la famille de polydisques $R(z, \delta)$ définis par $R(z, \delta)=\left\{\zeta \in \mathbb{C}^{2}, \zeta=\left(\zeta_{1}, \zeta_{2}\right),\left|\zeta_{1}\right| \leq \tau(z, \delta)\right.$ et $\left.\left|\zeta_{2}\right| \leq \delta\right\}$ pour définir dans $U$ la pseudo-distance $d_{0}(z, \delta)$ par

$$
d_{0}(z, \zeta)=\inf \{\delta>0, \zeta \in Q(z, \delta)\}
$$

où $Q(z, \delta)=\left\{\Phi_{z}(\zeta), \zeta \in R(z, \delta)\right\}$. Cette pseudo-distance n'étant définie que dans un voisinage du bord, on la prolonge à $\mathbb{C}^{2}$ à l'aide de la distance euclidienne. On considère $\psi \in \mathcal{C}^{\infty}\left(\mathbb{C}^{2}\right)$ telle que $\psi(z, \zeta)=1$ lorsque $|r(z)| \leq \varepsilon / 2$ et $|r(\zeta)| \leq \varepsilon / 2$ et $\psi(z, \zeta)=0$ lorsque $|r(z)| \geq \varepsilon$ ou $|r(\zeta)| \geq \varepsilon$.

Définition. Soient $z$ et $\zeta$ dans $\mathbb{C}^{2}$. On note

$$
d(z, \zeta)=\psi(z, \zeta) d_{0}(z, \zeta)+(1-\psi(z, \zeta))|z-\zeta|
$$

Cette pseudo-distance permet de définir la quantité $D(z, \zeta)$ suivante: pour tout point $z$ dans $\Omega$, on note $\delta(z)=\operatorname{dist}(z, \partial \Omega)$ et on pose

$$
D(z, \zeta)=d(\pi(z), \pi(\zeta))+\delta(\zeta)+\delta(z),
$$

où $\pi(z)$ et $\pi(\zeta)$ sont les projetés de $z$ et $\zeta$ sur $\partial \Omega$.

Dans le cas de la boule unité de $\mathbb{C}^{n}, B^{q}(z, \zeta)$ est aussi le noyau de Bergman à poids $B_{q^{\prime}}(z, \zeta)$ pour $q^{\prime}$ convenable. Pour les domaines pseudo-convexes de type fini de $\mathbb{C}^{2}$, on va construire un noyau reproduisant pour les fonctions holomorphes. Ce noyau $H_{n}(z, \zeta)$, qui joue le rôle de $B^{q}(z, \zeta)$, est obtenu à partir du noyau de $\operatorname{Bergman} B(z, \zeta)$ en faisant une dérivation selon un champ anti-holomorphe complexe normal. Le fait qu'il soit reproduisant pour la mesure $d V_{n}(z)=(-r(z))^{n} d V(z)$ découle d'une intégration par parties. On utilise les résultats de A. Nagel, J.-P. Rosay, E. Stein et S. Wainger [9] pour obtenir, pour $H_{n}(z, \zeta)$, les estimations asymptotiques suivantes: 
Proposition A. Soit $n \in \mathbb{N}$. Pour tout entier $l$, il existe $C(l)>0$ tel que

$$
\left|Y_{1} \ldots Y_{l} H_{n}(z, \zeta)\right| \leq C(l) D(z, \zeta)^{-2-n-n_{2}} \tau(z, D(z, \zeta))^{-2-n_{1}},
$$

où $Y_{1}, \ldots, Y_{l}$ sont des éléments de $\{\bar{N}, N, \bar{L}, L\}$ parmi lesquels il y a $n_{1}$ champs $L$ ou $\bar{L}$ et $n_{2}=l-n_{1}$ champs $N$ ou $\bar{N}$.

On note $H_{n}$ l'opérateur intégral défini à partir de $H_{n}(z, \zeta)$ par

$$
H_{n} f(z)=\int_{\Omega} H_{n}(z, \zeta) f(\zeta) d V_{n}(\zeta) \text { pour tout } f \in L^{1}(\Omega) .
$$

On pose $\mu(z)=\tau(z, \delta(z))$ lorsque $z \in \Omega \cap U$ et $\mu(z)=1$ sinon. Pour $\alpha>-1$, on note $d V_{\alpha}(z)=(-r(z))^{\alpha} d V(z)$. Pour $0<p<+\infty$, on pose $A^{p}\left(\mu(z)^{\beta} d V_{\alpha}\right)=L^{p}\left(\mu(z)^{\beta} d V_{\alpha}\right) \cap \mathcal{H}(\Omega)$ et on note $\|\cdot\|_{\alpha, \beta, p}$ sa norme. Comme $\Omega$ est un domaine de type fini $m$, il existe $c_{1}>0$ et $c_{2}>0$ telles que

$$
c_{1} \delta^{1 / 2} \leq \tau(z, \delta) \leq \delta^{1 / m} c_{2}
$$

pour tout $z$ dans $\Omega \cap U$ et pour tout $0<\delta<1[\mathbf{1}]$. On note $h$ la fonction définie par $h(x)=2$ si $x \leq 0$ et $h(x)=m$ si $x>0$. Les estimations données par la Proposition A et le critère de Schur permettent de montrer que $H_{n}$ se prolonge en un opérateur continu dans $L^{p}\left(\mu(z)^{\beta} d V_{\alpha}\right)$, quand $1<p<+\infty[\mathbf{6}]$ et $[\mathbf{1 0}]$.

Proposition B. Soient $1<p<+\infty$, $\alpha$ et $\beta$ tels que $1+\alpha+\frac{\beta}{h(\beta)}>0$. Il existe $n_{0}=n_{0}(\alpha, \beta, p)$ tel que pour $n>n_{0}$

$$
H_{n} \text { est un opérateur continu dans } L^{p}\left(\mu(\zeta)^{\beta} d V_{\alpha}\right) \text {. }
$$

Proposition C. Soient $0<p<+\infty$, $\alpha$ et $\beta$ tels que $1+\alpha+\frac{\beta}{h(\beta)}>0$. Il existe $n_{0}=n_{0}(\alpha, \beta, p)$ tel que pour $n>n_{0}$

$$
F(z)=\int_{\Omega} H_{n}(z, \zeta) F(\zeta) d V_{n}(\zeta) \text { pour tout } F \text { dans } A^{p}\left(\mu(\zeta)^{\beta} d V_{\alpha}\right)
$$

On utilise le noyau $H_{n}(z, \zeta)$ et la formule de représentation intégrale précédente pour montrer le théorème de décomposition atomique des espaces de Bergman. Bien que les espaces de Bergman classiques soient les espaces $A^{p}\left(d V_{\alpha}\right), \alpha>-1$ [3] et [4], le théorème suivant donne la décomposition des espaces $A^{p}\left(\mu(z)^{\beta} d V_{\alpha}\right)$, lorsque $(-r(\zeta))^{\alpha} \mu(z)^{\beta}$ est un poids intégrable car ils interviennent dans la caractérisation des opérateurs de Hankel. 
Théorème D. Soient $0<p<+\infty, \alpha, \beta$ tels que $1+\alpha+\frac{\beta}{h(\beta)}>0$. Il existe une suite $K_{i}(z)$ dans $A^{p}\left(\mu(\zeta)^{\beta} d V_{\alpha}\right)$ telle que pour tout $F$ dans $A^{p}\left(\mu(z)^{\beta} d V_{\alpha}\right)$, il existe $\left(\lambda_{i}\right) \in \ell^{p}$ telle que

(i) $F(z)=\sum_{i} \lambda_{i} K_{i}(z)$,

(ii) $1 / C\left(\sum_{i}\left|\lambda_{i}\right|^{p}\right)^{1 / p} \leq\|F\|_{\alpha, \beta, p} \leq C\left(\sum_{i}\left|\lambda_{i}\right|^{p}\right)^{1 / p}$.

Dans le Théorème $\mathrm{D}$, les fonctions $K_{i}$ sont définies à partir du noyau $H_{n}(z, \zeta)$ pour un indice $n$ assez grand. Suivant R. Coifman et R. Rochberg, on démontre le Théorème $\mathrm{D}$ en approchant la fonction $F$. Pour cela, on discrétise la relation 1.3 pour obtenir une somme de Riemann adaptée. On construit une suite de points $\left(w_{i}\right)$ liée à un recouvrement de Whitney du domaine pour la pseudo-distance $d$. Lorsque le recouvrement est constitué par des domaines suffisamment petits, la fonction $G$, obtenue en considérant la somme de Riemann associée au recouvrement, vérifie $\|F-G\|_{\alpha, \beta, p} \leq 1 / 2\|F\|_{\alpha, \beta, p}$. On conclut par un argument d'analyse fonctionnelle.

\section{Projecteurs à poids}

Le but de cette partie est de construire le noyau $H_{n}(z, \zeta)$ qui est reproduisant pour les éléments de $A^{p}\left(\mu(z)^{\beta} d V_{\alpha}\right)$ puis d'établir des estimations asymptotiques. Le noyau $H_{n}(z, \zeta)$ est obtenu à partir du noyau de Bergman à l'aide d'intégrations par parties. Pour tout point $p$ sur $\partial \Omega$, on note $L$ le champ holomorphe tangent en $p$ à $\partial \Omega$ défini par

$$
L=\frac{\partial r}{\partial z_{2}} \frac{\partial}{\partial z_{1}}-\frac{\partial r}{\partial z_{1}} \frac{\partial}{\partial z_{2}}
$$

et $N$ le champ holomorphe normal

$$
N=2\left(\frac{\partial r}{\partial \bar{z}_{1}} \frac{\partial}{\partial z_{1}}+\frac{\partial r}{\partial \bar{z}_{2}} \frac{\partial}{\partial z_{2}}\right) .
$$

En particulier $|N r|=1$ sur $\partial \Omega$.

Pour construire $H_{n}(z, \zeta)$, on part du fait que le noyau de Bergman est un noyau reproduisant pour les éléments de $A^{2}(\Omega)$. Ainsi $F \in A^{2}(\Omega)$ vérifie

$$
F(z)=\int_{\Omega} B(z, \zeta) F(\zeta) d V(\zeta)
$$


Comme $|\nabla r|=1$ sur $\partial \Omega$, il existe deux fonctions $a(\zeta)$ et $b(\zeta)$ dans $\mathcal{C}^{\infty}(\bar{\Omega})$ telles que $1=a(\zeta) \bar{N} r(\zeta)+b(\zeta) r(\zeta)$. On obtient le noyau $H_{n}(z, \zeta)$ par récurrence. On pose $H_{0}(z, \zeta)=B(z, \zeta)$ et on obtient $H_{n}(z, \zeta)$ à partir de $H_{n-1}(z, \zeta)$ en posant, pour $F$ dans $\mathcal{C}^{\infty}(\bar{\Omega}) \cap \mathcal{H}(\Omega)$,

$$
\begin{aligned}
F(z)= & \int_{\Omega} H_{n-1}(z, \zeta) F(\zeta) d V_{n-1}(\zeta) \\
= & -\frac{1}{n} \int_{\Omega} a(\zeta) H_{n-1}(z, \zeta) F(\zeta) \bar{N}\left(-r(\zeta)^{n}\right) d V(\zeta) \\
& -\int_{\Omega} H_{n-1}(z, \zeta) b(\zeta) F(\zeta) d V_{n}(\zeta) \\
= & I_{1}+I_{2} .
\end{aligned}
$$

Par intégration par parties,

$$
\begin{array}{r}
I_{1}=\frac{1}{n} \int_{\Omega}\left(a(\zeta) \bar{N}_{\zeta} H_{n-1}(z, \zeta)+\left(\bar{N}_{\zeta} a(\zeta)+\frac{1}{4} a(\zeta) \Delta r(\zeta)\right) H_{n-1}(z, \zeta)\right) \\
F(\zeta) d V_{n}(\zeta) .
\end{array}
$$

On obtient alors

$H_{n}(z, \zeta)=\frac{a(\zeta)}{n} \bar{N}_{\zeta} H_{n-1}(z, \zeta)+\left(\frac{\bar{N}_{\zeta} a(\zeta)+\Delta r(\zeta) / 4}{n}-b(\zeta)\right) H_{n-1}(z, \zeta)$.

Pour montrer la Proposition A, on commence par noter qu'il existe $a_{0}, \ldots, a_{n}$ dans $\mathcal{C}^{\infty}(\bar{\Omega})$ telles que

$$
H_{n}(z, \zeta)=\sum_{i=0}^{n} a_{i}(\zeta) \bar{N}^{i} B(z, \zeta)
$$

On utilise le résultat suivant $[\mathbf{9}]$ : Soient $Y_{1}, \ldots, Y_{l}$ des éléments de $\{\bar{N}, N, \bar{L}, L\}$. On suppose qu'il y a $n_{1}$ champs $L$ ou $\bar{L}$ et $n_{2}=l-n_{1}$ champs $N$ ou $\bar{N}$. Il existe $C(l)>0$ tel que

$$
\left|Y_{1} \ldots Y_{l} B(z, \zeta)\right| \leq \frac{C(l) D(z, \zeta)^{-n_{2}} \tau(z, D(z, \zeta))^{-n_{1}}}{\operatorname{Vol}(Q(z, D(z, \zeta)))} .
$$

\section{Propriétés de l'opérateur $H_{n}$}

Dans cette partie, on montre que $H_{n}$ se prolonge en un opérateur continu dans certains $L^{p}$ pour $1<p<+\infty$. Dans le cas $n=0$, il est bien connu que le projecteur de Bergman est un opérateur continu dans $L^{p}, 1<p<+\infty[\mathbf{9}]$. Pour les opérateurs $H_{n}$, la démonstration de la Proposition B repose sur le critère de Schur qui permet de donner une condition suffisante pour qu'un opérateur défini à partir d'un noyau positif soit borné dans $L^{p}(\Omega)$ pour $1<p<+\infty[\mathbf{3}]$ et $[\mathbf{1 0}]$. On va utiliser ce critère avec la fonction $g(\zeta)=\delta(\zeta)^{a}$ pour $a$ dans $]-1,0[$ convenablement choisi. On commence par prouver le résultat suivant: 
Lemme 3.1. Pour tous a et $b$ tels que $0<1+a+\frac{b}{h(b)}$ et $a-n+\frac{b}{h(-b)}<$ 0 , il existe $C=C(a, b)>0$ tel que pour tout $z \in \Omega$,

$$
\int_{\Omega}\left|H_{n}(z, \zeta)\right| \delta(\zeta)^{a} \mu(\zeta)^{b} d V(\zeta) \leq C \delta(z)^{a-n} \mu(z)^{b} .
$$

Démonstration: Il suffit de considérer le cas où $z \in U \cap \Omega$. Le lemme repose sur un découpage de $\Omega$ en tentes $B_{l}=Q\left(\pi(z), 2^{l} \delta(z)\right) \cap \Omega$. Dans ce cas

$$
\begin{aligned}
\int_{\Omega}\left|H_{n}(z, \zeta)\right| \mu(\zeta)^{\beta} \delta(\zeta)^{a} d V(\zeta) \leq & \int_{B_{1}}\left|H_{n}(z, \zeta)\right| \mu(\zeta)^{\beta} \delta(\zeta)^{a} d V(\zeta) \\
& +\sum_{l=2}^{+\infty} \int_{B_{l} \backslash B_{l-1}}\left|H_{n}(z, \zeta)\right| \mu(\zeta)^{\beta} \delta(\zeta)^{a} d V_{n}(\zeta)
\end{aligned}
$$

Sur $B_{1}$, on utilise le fait que $\left|H_{n}(z, \zeta)\right| \leq\left|H_{n}(z, \pi(z))\right| \leq C \mu(z)^{-2} \delta(z)^{-2-n}$ pour obtenir

$$
\int_{B_{1}}\left|H_{n}(z, \zeta)\right| \delta(\zeta)^{a} \mu(\zeta)^{b} d V(\zeta) \leq C \delta(z)^{a-n} \mu(z)^{b}
$$

Pour estimer les autres termes, il suffit de noter que pour tout $\zeta$ dans $B_{l} \backslash B_{l-1}, \delta(\zeta) \simeq 2^{l} \delta(z)$ et que, d'après la relation $1.2, c_{1} 2^{l / m} \mu(\zeta)<$ $\mu\left(\pi(\zeta), 2^{l} \delta(\zeta)\right)<c_{2} 2^{l / 2} \mu(\zeta)$. On obtient

$$
\begin{aligned}
\int_{B_{l} \backslash B_{l-1}}\left|H_{n}(z, \zeta)\right| \delta(\zeta)^{a} \mu(\zeta)^{b} d V_{n}(\zeta) & \leq C \delta(z)^{a-n} \mu(z)^{b} 2^{(a-n) l+\frac{b}{h(-b)}} \frac{\operatorname{Vol}\left(B_{l}\right)}{\operatorname{Vol}\left(B_{l-1}\right)} \\
& \leq C \delta(z)^{a-n} \mu(z)^{b} 2^{(a-n) l+\frac{b l}{h(-b)}}
\end{aligned}
$$

Soient $p$ et $p^{\prime}$ tels que $1 / p+1 / p^{\prime}=1$. Pour montrer que $H_{n}$ est un opérateur borné dans $L^{p}\left(\mu(z)^{\beta} d V_{\alpha}\right)$, on montre que l'opérateur $\tilde{H}_{n}$ défini par

$$
\tilde{H}_{n} f(z)=\int_{\Omega}\left|H_{n}(z, \zeta)\right|\left(\frac{\delta(\zeta)^{n-\alpha} \mu(\zeta)^{-\beta}}{\delta(z)^{n-\alpha} \mu(z)^{-\beta}}\right)^{1 / p} f(\zeta) d V_{n}(\zeta)
$$

est borné dans $L^{p}\left(d V_{n}\right)$. Pour cela on utilise le critère de Schur: il s'agit alors de montrer qu'il existe $g(z)=\delta(z)^{a}$ tel que

$$
\begin{aligned}
& \left(\delta(z)^{\alpha-n} \mu(z)^{\beta}\right)^{1 / p} \int_{\Omega}\left|H_{n}(z, \zeta)\right| \delta(\zeta)^{n+(n-\alpha) / p+p^{\prime} a} \mu(\zeta)^{-\beta / p} d V(\zeta) \leq C g(z)^{p^{\prime}} \\
& \left(\delta(\zeta)^{n-\alpha} \mu(\zeta)^{-\beta}\right)^{1 / p} \int_{\Omega}\left|H_{n}(z, \zeta)\right| \delta(z)^{n+(\alpha-n) / p+p a} \mu(z)^{\beta / p} d V(z) \leq C g(\zeta)^{p}
\end{aligned}
$$


Les deux conditions sont réalisées en vertu du Lemme 3.1 est choisi tel que

$$
(n-\alpha) / p+\frac{-\beta / p}{h(\beta)}<-p^{\prime} a<n+1+(n-\alpha) / p-\frac{\beta / p}{h(-\beta)}
$$

et

$$
(\alpha-n) / p+\frac{\beta / p}{h(-\beta)}<-p a<n+1+(\alpha-n) / p+\frac{\beta / p}{h(\beta)}
$$

Pour que $a$ existe, il est nécessaire et suffisant que $1+\alpha+\frac{\beta}{h(\beta)}>0$ et $n$ verifie $n>n_{1}(\alpha, \beta, p)=\frac{1}{p}\left(\alpha+\frac{\beta}{h(-\beta)}\right)+\frac{1}{p}-1$.

Soit $f$ dans $L^{p}\left(\mu(z)^{\beta} d V_{\alpha}\right)$. La fonction $f_{1}(\zeta)=f(\zeta) \delta(\zeta)^{(\alpha-n) / p} \mu(\zeta)^{\beta / p}$ est dans $L^{p}\left(d V_{n}\right)$ et $\|f\|_{\alpha, \beta, p}=\left\|f_{1}\right\|_{n, 0, p}$. L'opérateur $\tilde{H}_{n}$ étant continu dans $L^{p}\left(d V_{n}\right)$, il existe $C>0$ tel que $\left\|\tilde{H}_{n} f_{1}\right\|_{n, 0, p} \leq C\left\|f_{1}\right\|_{n, 0, p}$. Il reste à noter que $\|H f\|_{\alpha, \eta, p} \leq\left\|\tilde{H}_{n} f_{1}\right\|_{n, 0, p}$ pour achever la démonstration de la Proposition B.

\section{Formule de représentation intégrale}

Dans cette partie, on donne une condition sur $n$ pour que $H_{n}(\zeta, z)$ soit un noyau reproduisant pour les fonctions de $A^{p}\left(\mu(z)^{\beta} d V_{\alpha}\right)$. Pour cela on utilise le lemme d'inclusion qui suit mais qui n'est probablement pas optimal.

Lemme 4.1. Soient $0<p<+\infty$, $\alpha$ et $\beta$ tels que $1+\alpha+\frac{\beta}{h(\beta)}>0$. Il existe $n_{2}=n_{2}(\alpha, \beta, p)$ tel que pour $n \in \mathbb{N}$ tel que $n>n_{2}$,

$$
A^{p}\left(\mu(\zeta)^{\beta} d V_{\alpha}\right) \subseteq A^{2}\left(d V_{n}\right)
$$

Démonstration du lemme: Lorsque $p>2$, l'inclusion est une conséquence de la relation de Hölder et $n_{2}=\frac{2}{p}\left(\alpha+\frac{\beta}{h(-\beta)}\right)+\frac{2}{p}-1$. Pour $0<p \leq 2$, le domaine $\Omega$ étant un domaine de nature homogène au sens de R. Coifman et G. Weiss, on considère un recouvrement de Whitney de $\Omega$ par des domaines $Q(z, \theta \delta(z)), \theta>0$ suffisamment petit de sorte que pour tout $z, Q(z, \theta \delta(z)) \subset \Omega[\mathbf{2}]$. Pour tout élément $Q_{i}$ du recouvrement, on note $z_{i}$ le centre $Q_{i}$. On pose aussi $\bar{Q}_{i}=Q\left(z_{i}, C_{0} \theta \delta\left(z_{i}\right)\right)$. Comme les $Q_{i}$ forment un recouvrement de Whitney, on choisit $C_{0}$ pour que les $\tilde{Q}_{i}=$ $Q\left(z_{i}, \theta \delta\left(z_{i}\right) / C_{0}\right)$ soient deux à deux disjoints. Soit $F \in A^{p}\left(\mu(z)^{\beta} d V_{\alpha}\right)$. Pour tout point $z$ dans $Q_{i}$, on part de l'inégalité suivante qui découle de la sous-harmonicité de $|F|^{p}$ :

$$
|F(z)|^{p} \leq \frac{C}{\operatorname{Vol}\left(\bar{Q}_{i}\right)} \int_{\bar{Q}_{i}}|F(\zeta)|^{p} d V(\zeta) .
$$


On obtient alors

$$
\begin{aligned}
\|F\|_{n, 0,2}^{2} & \leq \sum_{i} \int_{Q_{i}}|F(\zeta)|^{2} \delta(\zeta)^{n} d V(\zeta) \\
& \leq \sum_{i} \delta\left(z_{i}\right)^{n-2 \alpha / p} \mu\left(z_{i}\right)^{-2 \beta / p} \operatorname{Vol}\left(Q_{i}\right)^{1-2 / p}\left(\int_{\bar{Q}_{i}}|F(\zeta)|^{p} \mu(\zeta)^{\beta} d V_{\alpha}(\zeta)\right)^{2 / p}
\end{aligned}
$$

Les domaines $\bar{Q}_{i}$ étant presques disjoints, $\|F\|_{n, 0,2} \leq C\|F\|_{\alpha, \beta, p}$ dès que

$$
n \geq n_{2}=\frac{2}{p}\left(\alpha+\frac{\beta}{h(p-2-\beta)}\right)+2\left(\frac{2}{p}-1\right)\left(1+\frac{1}{h(p-2-\beta)}\right) .
$$

Citons le corollaire suivant:

Corollaire 4.2. Soient $0<p<+\infty, \alpha, \beta$ tels que $1+\alpha+\frac{\beta}{h(\beta)}>0$, $n$ entier tel que $n>n_{2}$. Toute fonction $F$ de $A^{p}\left(\mu(z)^{\beta} d V_{\alpha}\right)$ vérifie

$$
F(z)=\int_{\Omega} H_{n}(z, \zeta) F(\zeta) d V_{n}(\zeta)
$$

Démonstration du corollaire: On note $\rho(z)=-(-r(z))^{1 / k}$, où $k$ est un entier assez grand de sorte que le Théorème 1 de [5] soit vérifié. Un résultat de Ligocka assure que l'opérateur de Bergman à poids $B_{s}$ associé à la mesure $(-\rho(z))^{s} d V(z)$ est continu dans $\mathcal{C}^{\infty}(\bar{\Omega})[7]$. En particulier on en déduit que $\mathcal{C}^{\infty}(\bar{\Omega}) \cap \mathcal{H}(\Omega)$ est dense dans $A^{2}\left(d V_{n}\right)$. D'après le lemme précédent, $F$ est dans $A^{2}\left(d V_{n}\right)$. Soit $F_{\nu}$ une suite d'éléments de $\mathcal{C}^{\infty}(\bar{\Omega}) \cap \mathcal{H}(\Omega)$ approchant $F$. Alors, pour tout $\nu, H_{n} F_{\nu}=F_{\nu}$. On obtient la formule de représentation intégrale en notant que $H_{n}$ est continu dans $L^{2}\left(d V_{n}\right)$.

\section{Décomposition atomique}

Pour établir le Théorème D pour, on va utiliser la formule de représentation intégrale donnée par le Corollaire 4.2 pour approcher une fonction $F$ de $A^{p}\left(\mu(z)^{\beta} d V_{\alpha}\right)$. On considère un recouvrement de Whitney de $\Omega$ par des domaines $Q_{i}$ de la forme $Q(w, \eta \theta \delta(w)), 0<\eta<1$ et on note $w_{i}$ les centres de ces domaines. Cette suite, selon la terminologie de $\mathrm{R}$. Coifman et R. Rochberg, est appelée un $\eta$-réseau sur $\Omega$. On pose $\tilde{Q}_{i}=Q\left(w_{i}, \eta \theta \delta\left(w_{i}\right) / C_{0}\right)$ et on définit les fonctions $K_{i}(z)$ par

$$
K_{i}(z)=\delta\left(w_{i}\right)^{n-\alpha / p} \mu\left(w_{i}\right)^{-\beta / p} \operatorname{Vol}\left(Q\left(w_{i}, \theta \delta\left(w_{i}\right)\right)^{1-1 / p} H_{n}\left(z, w_{i}\right),\right.
$$


où $n$ est un entier tel que $n>n_{2}$. La Proposition $\mathrm{A}$ et un découpage de $\Omega$ en tentes $B_{l}$ perment de montrer que $K_{i}$ est un élément de $A^{p}\left(\mu(z)^{\beta} d V_{\alpha}\right)$ dès que

$$
\begin{aligned}
n>n_{3}(\alpha, \beta, p)= & \frac{1}{p}\left(\alpha+\frac{\beta}{h(2(p-1)-\beta)}\right) \\
& +2\left(\frac{1}{p}-1\right)\left(1+\frac{1}{h(2(p-1)-\beta)}\right) .
\end{aligned}
$$

On construit une fonction $G$ qui approche la fonction $F$ de la façon suivante:

Proposition 5.1. Soient $0<p<+\infty, \alpha, \beta$ tels que $1+\alpha+\frac{\beta}{h(\beta)}>$ 0 et $n \in \mathbb{N}, n>n_{2}$. Pour tout $F \in A^{p}\left(\mu(z)^{\beta} d V_{\alpha}\right)$, il existe $G \in$ $A^{p}\left(\mu(z)^{\beta} d V_{\alpha}\right)$ telle que

(i) $G(z)=\sum_{i} \nu_{i} K_{i}(z)$, où $\left(\nu_{i}\right)$ est une suite de $\ell^{p}$,

(ii) $\|F-G\|_{\alpha, \beta, p} \leq \frac{1}{2}\|F\|_{\alpha, \beta, p}$.

Démonstration: Les domaines $\tilde{Q}_{i}$ étant deux à deux disjoints, on associe à la suite $\left(w_{i}\right)$ un recouvrement de $\Omega$ par des domaines deux à deux disjoints $E_{i}$ dont on sait estimer le volume. On pose $E_{0}=Q_{0} \backslash\left(\bigcup_{j=1}^{+\infty} \tilde{Q}_{i}\right)$ et, pour tout indice $i$, on note

$$
E_{i}=Q_{i} \backslash\left(\left(\bigcup_{j=0}^{i-1} E_{i}\right) \cup\left(\bigcup_{j=i+1}^{+\infty} \tilde{Q}_{i}\right)\right)
$$

Il est immédiat que $\tilde{Q}_{i} \subseteq E_{i} \subseteq Q_{i}$, que $\bigcup_{i=0}^{+\infty} E_{i}=\Omega$ et que $E_{i} \cap E_{j}=$ $\emptyset$ si $i \neq j$. On obtient la fonction $G$ en discrétisant la formule de représentation intégrale donnée par le Corollaire 4.2. Ainsi

$$
G(z)=\sum_{i} F\left(w_{i}\right) \operatorname{Vol}_{n}\left(E_{i}\right) H_{n}\left(z, w_{i}\right)
$$

Dans ce cas $\nu_{i}=\frac{F\left(w_{i}\right) \operatorname{Vol}_{n}\left(E_{i}\right)}{\delta\left(w_{i}\right)^{n-\alpha / p} \mu\left(w_{i}\right)^{-\beta / p} \operatorname{Vol}\left(E_{i}\right)^{1-1 / p}}$. On montre que la suite $\left(\nu_{i}\right)$ est un élément de $\ell^{p}$ en notant que, pour tout indice $i, \operatorname{Vol}_{n}\left(E_{i}\right) \simeq$ 
$\delta\left(w_{i}\right)^{n} \operatorname{Vol}\left(E_{i}\right)$ et que $\left|\nu_{i}\right|^{p} \leq C \operatorname{Vol}\left(\tilde{Q}_{i}\right)\left|F\left(w_{i}\right)\right|^{p} \delta\left(w_{i}\right)^{\alpha} \mu\left(w_{i}\right)^{\beta}$. La sous harmonicité de $|F|^{p}$ entraîne

$$
\sum_{i}\left|\nu_{i}\right|^{p} \leq C \sum_{i} \int_{\tilde{Q}}|F(\zeta)|^{p} \mu(\zeta)^{\beta} d V_{\alpha}(\zeta) \leq C\|F\|_{\alpha, \beta, p}^{p}
$$

car les $\tilde{Q}_{i}$ sont deux à deux disjoints. Afin d'estimer $\|F-G\|_{\alpha, \beta, p}$ on écrit

$$
\begin{aligned}
|F(z)-G(z)| \leq & \sum_{i}\left|H_{n}\left(z, w_{i}\right)\right| \int_{E_{i}}\left|F(\zeta)-F\left(w_{i}\right)\right| d V_{n}(\zeta) \\
& +\sum_{i} \int_{E_{i}}|F(\zeta)|\left|H_{n}\left(z, w_{i}\right)-H_{n}(z, \zeta)\right| d V_{n}(\zeta) \\
\leq & S_{1}+S_{2} .
\end{aligned}
$$

Pour estimer $S_{1}$ et $S_{2}$ en fonction de $\eta^{1 / m}$, on commence par montrer le résultat suivant:

Lemme 5.2. Il existe $\gamma \geq 1$ et $C>0$ tels que pour tout $z$ dans $U$, $w \in Q(z, \theta \delta(z))$ et $\eta$ assez petit de sorte que $Q(w, \eta \theta \delta(w)) \subset Q(z, \theta \delta(z))$,

(i) $\sup _{\zeta \in Q(w, \eta \theta \delta(w))}|F(\zeta)-F(w)|^{p} \leq \frac{C \eta^{p / m}}{\operatorname{Vol}(Q(z, \theta \delta(z)))} \int_{\bar{Q}}|F(\zeta)|^{p} d V(\zeta)$,
$\quad$ où $\bar{Q}=Q(z, \gamma \theta \delta(z))$,
(ii) $\sup _{\zeta \in Q(w, \eta \theta \delta(w))}\left|H_{n}(z, \zeta)-H_{n}(z, w)\right| \leq C \eta^{1 / m} D(z, w)^{-2-n} \tau(z, D(z, w))^{-2} \cdot$

Démonstration: Pour obtenir (i), on rappelle que si on considère $P_{1}$ un polydisque de $\mathbb{C}^{2}$ de centre 0 et de rayon $\left(R_{1}, R_{2}\right)$ contenant un polydisque $P_{2}$ de centre 0 et de rayon $\left(r_{1}, r_{2}\right)$ de mêmes directions que $P_{1}$ et que l'on suppose que $0<r_{1} \leq \xi R_{1}$ et $0<r_{2} \leq \xi R_{2}$ où $0<\xi<1 / 4$, il existe $C>0$, indépendant de $\xi$ tel que pour toute fonction $f$ holomorphe dans un voisinage de $\bar{P}_{1}$,

$$
\sup _{z \in P_{2}}|f(z)-f(0)|^{p} \leq \frac{C \xi^{p}}{\operatorname{Vol}\left(P_{1}\right)} \int_{P_{1}}|f(\zeta)|^{p} d V(\zeta) .
$$

Soit $w \in U$. Pour majorer $|F(\zeta)-F(w)|$ sur $Q(w, \eta \theta \delta(w)))$, on considère $\Phi_{w}$, le changement de variable associé au point $w$ et on définit la fonction $F_{w}$ sur $R(w, \theta \delta(w))$ par $F_{w}(u)=F\left(\Phi_{w}(u)\right)$. La relation 5.7 avec les polydisques $R(w, \eta \theta \delta(w))$ et $R(w, \theta \delta(w))$ entraîne

$\sup _{R(w, \eta \theta \delta(w))}\left|F_{w}(u)-F_{w}(0)\right|^{p} \leq \frac{C \eta^{p / m}}{\operatorname{Vol}(R(w, \theta \delta(w)))} \int_{R(w, \theta \delta(w))}\left|F_{w}(\zeta)\right|^{p} d V(\zeta)$. 
Quitte à réduire $\theta$, il existe $\gamma \geq 1$ tel que $\Phi_{w}((w, \theta \delta(w))) \subseteq Q(z, \gamma \theta \delta(z)) \subset$ $\Omega$, ce qui donne l'estimation cherchée.

Pour démontrer la partie (ii), on utilise la relation 2.5 pour écrire

$$
\begin{aligned}
\left|H_{n}(z, \zeta)-H_{n}(z, w)\right| \leq & \sum_{l=0}^{n}\left|\bar{N}^{l} B(z, w)\right|\left|a_{l}(\zeta)-a_{l}(w)\right| \\
& +\sum_{l=0}^{n}\left|a_{l}(\zeta)\right|\left|\bar{N}^{l} B(z, \zeta)-\bar{N}^{l} B(z, w)\right| .
\end{aligned}
$$

La majoration de la première somme découle de la Proposition $\mathrm{A}$ et du fait que les $a_{l}$ ont des dérivées bornées. Pour la seconde, on utilise le résultat suivant:

Lemme 5.3. Soient $l$ dans $\mathbb{N}$. Il existe $C(l)>0$ tel que pour tout $z$ dans $U, w$ dans $Q(z, \theta \delta(z))$ et $\eta>0$ assez petit tels que $Q(w, \eta \theta \delta(w)) \subset$ $Q(z, \theta \delta(z))$,

$\sup _{\zeta \in Q(w, \eta \theta \delta(w))}\left|\bar{N}^{l} B(z, \zeta)-\bar{N}^{l} B(z, w)\right| \leq C(l) \eta^{1 / m} D(z, w)^{-l-2} \tau(z, D(z, w))^{-2}$.

Démonstration du lemme: On définit la fonction $b_{l}$ par $b_{l}(u)=$ $\bar{N}^{l} B\left(z, \Phi_{w}(u)\right)$. Si on note $u=\left(u_{1}, u_{2}\right)$, comme $\left|b_{l}(u)-b_{l}(0)\right| \leq$ $\left|b_{l}\left(u_{1}, u_{2}\right)-b_{l}\left(u_{1}, 0\right)\right|+\left|b_{l}\left(u_{1}, 0\right)-b_{l}(0)\right|$, il s'agit d'estimer

$$
\sup _{R(w, \eta \theta \delta(w))}\left|b_{l}\left(u_{1}, u_{2}\right)-b_{l}\left(u_{1}, 0\right)\right| \text { et } \sup _{R(w, \eta \theta \delta(w))}\left|b_{l}\left(u_{1}, 0\right)-b_{l}(0)\right| .
$$

Le premier terme est majoré par

$$
\eta \delta(w) \sup _{R(w, \eta \theta \delta(w))}\left(\left|\frac{\partial b_{l}(u)}{\partial u_{1}}\right|+\left|\frac{\partial b_{l}(u)}{\partial \bar{u}_{1}}\right|\right) .
$$

D'après la Proposition A, si on note $v=\Phi_{w}(u)$, alors

$$
\left|\frac{\partial b_{l}(u)}{\partial u_{2}}\right|+\left|\frac{\partial b_{l}(u)}{\partial \bar{u}_{2}}\right| \leq D(z, u)^{-3-l} \tau(z, D(z, v))^{-2} .
$$

Il suffit ensuite de noter que dans $Q(z, \theta \delta(z)), D(z, v) \simeq D(z, w)$ et $\tau(z, v) \simeq \tau(z, w)$.

Pour majorer le second terme, il faut estimer

$$
\tau(w, \eta \delta(w)) \sup _{R(w, \eta \theta \delta(w))}\left(\left|\frac{\partial b_{l}(u)}{\partial u_{2}}\right|+\left|\frac{\partial b_{l}(u)}{\partial \bar{u}_{2}}\right|\right) .
$$


On procède de manière identique en utilisant cette fois le fait que, d'après la Proposition A,

$$
\left|\frac{\partial b_{l}(u)}{\partial u_{2}}\right|+\left|\frac{\partial b_{l}(u)}{\partial \bar{u}_{2}}\right| \leq C D(z, u)^{-2-l} \tau(z, D(z, v))^{-3} .
$$

Pour majorer $S_{1}$ et $S_{2}$ par $\|F\|_{\alpha, \beta, p}$, on définit le noyau positif $C_{n}(z, \zeta)$ par

$$
\begin{array}{ll}
C_{n}(z, \zeta)=\tau(z, D(z, \zeta))^{-2} D(z, \zeta)^{-2-n} & \text { si } z \text { et } \zeta \text { dans } U \cap \Omega \\
C_{n}(z, \zeta)=1 & \text { sinon. }
\end{array}
$$

Ceci permet de définir, pour tout $i$, la fonction $L_{i}(z)$ par

$$
L_{i}(z)=\delta\left(w_{i}\right)^{n-\alpha / p} \mu\left(w_{i}\right)^{-\beta / p} \operatorname{Vol}\left(Q\left(w_{i}, \theta \delta\left(w_{i}\right)\right)^{1-1 / p} C_{n}\left(z, w_{i}\right) .\right.
$$

Comme pour $K_{i}$, il existe $C>0$ tel que pour tout $i,\left\|L_{i}\right\|_{\alpha, \beta, p} \leq C$ pour $n>n_{3}$. Il est immédiat qu'il existe $C>0$ indépendant de $\eta$ et de $F$ tel que

$$
S_{2} \leq C \eta^{1 / m} \sum_{i}\left(\int_{\bar{Q}_{i}}|F(\zeta)|^{p} \mu(\zeta)^{\beta} d V_{\alpha}(\zeta)\right)^{1 / p} L_{i}(z)
$$

où $\bar{Q}_{i}=Q\left(w_{i}, \gamma \theta \delta\left(w_{i}\right)\right)$.

On obtient une majoration identique pour $S_{1}$, en notant que dans $Q(z, \gamma \theta \delta(z)), \delta(\zeta) \simeq \delta(w)$ et $\mu(\zeta) \simeq \mu(w)$. Ceci donne, compte tenu du Lemme 5.2,

$$
\begin{aligned}
\int_{E_{i}}|F(\zeta)|-F\left(w_{i}\right) \mid d V_{n}(\zeta) \leq \frac{C \eta^{1 / m} \delta\left(w_{i}\right)^{n-\alpha / p} \mu\left(w_{i}\right)^{-\beta / p}}{\operatorname{Vol}\left(Q\left(w_{i}, \theta \delta\left(w_{i}\right)\right)\right)^{1 / p-1}} & \left(\int_{\bar{Q}_{i}}|F(\zeta)|^{p} \mu(\zeta)^{\beta} d V_{\alpha}(\zeta)\right)^{1 / p}
\end{aligned}
$$

D'après la Proposition A, $\left|H_{n}(z, \zeta)\right| \leq C C_{n}\left(z, w_{i}\right)$ pour tout $\zeta$ dans $E_{i}$, donc

$$
S_{1} \leq C \eta^{1 / m} \sum_{i}\left(\int_{\bar{Q}_{i}}|F(\zeta)|^{p} \mu(\zeta)^{\beta} d V_{\alpha}(\zeta)\right)^{1 / p} L_{i}(z) .
$$

On utilise alors le résultat suivant pour conclure:

Proposition 5.4. Soient $0<p<+\infty, \alpha, \beta$ tels que $1+\alpha+\frac{\beta}{h(\beta)}>0$ et

$$
\begin{aligned}
& n>n_{3} \text { si } 0<p \leq 1 \\
& n>n_{1} \text { si } 1<p<+\infty
\end{aligned}
$$


Il existe $C>0$ tel que pour tout suite $\left(\lambda_{i}\right)$ de $\ell^{p}$,

$$
\left\|\sum_{i} \lambda_{i} L_{i}\right\|_{\alpha, \beta, p} \leq C\left(\sum_{i}\left|\lambda_{i}\right|^{p}\right)^{1 / p} .
$$

Démonstration de la proposition: Dans ce cas $0<p \leq 1$, il suffit de remarquer que

$$
\left\|\sum_{i} \lambda_{i} L_{i}\right\|_{\alpha, \beta, p}^{p} \leq C \sum_{i}\left|\lambda_{i}\right|^{p}\left\|L_{i}\right\|_{p, \alpha, \beta}^{p} \leq C \sum_{i}\left|\lambda_{i}\right|^{p} .
$$

Pour $1<p<+\infty$. On définit $k(z)$ sur $\Omega$ par

$$
k(z)=\sum_{i}\left|\lambda_{i}\right| \delta\left(w_{i}\right)^{-\alpha / p} \mu\left(w_{i}\right)^{-\beta / p} \operatorname{Vol}\left(E_{i}\right)^{-1 / p} \mathbb{I}_{E_{i}},
$$

de sorte que $\|k\|_{\alpha, \beta, p} \simeq\left(\sum_{i}\left|\lambda_{i}\right|^{p}\right)^{1 / p}$. Comme $\left|H_{n}(z, \zeta)\right| \leq C C_{n}\left(z, w_{i}\right)$ sur $Q_{i}$, il existe $C>0$ tel que

$$
\sum_{i}\left|\lambda_{i}\right| \delta\left(w_{i}\right)^{n-\alpha / p} \mu\left(w_{i}\right)^{-\beta / p} \operatorname{Vol}\left(\tilde{Q}_{i}\right)^{1-1 / p} C_{n}\left(z, w_{i}\right) \leq C T_{n} k(z)
$$

où $T_{n}$ est l'opérateur intégral associé au noyau $C_{n}(z, \zeta)$. On obtient

$$
\begin{aligned}
\left|\sum_{i} \lambda_{i} L_{i}(z)\right| & \leq \sum_{i}\left|\lambda_{i}\right| \delta\left(w_{i}\right)^{n-\alpha / p} \mu\left(w_{i}\right)^{-\beta / p} \operatorname{Vol}\left(\tilde{Q}_{i}\right)^{1-1 / p} C_{n}\left(z, w_{i}\right) \\
& \leq C T_{n} k(z) .
\end{aligned}
$$

D'après la Proposition $\mathrm{B}, T_{n}$ est un opérateur continu dans $L^{p}\left(\mu(z)^{\beta} d V_{\alpha}\right)$ pour $n>n_{1}$ donc

$$
\left\|\sum_{i} \lambda_{i} L_{i}\right\|_{\alpha, \beta, p} \leq\left\|T_{n} k\right\|_{p, \alpha, \beta} \leq C\|k\|_{\alpha, \beta, p}=C\left(\sum_{i}\left|\lambda_{i}\right|^{p}\right)^{1 / p} .
$$

Lorsque l'on applique la Proposition 5.4 à la fonction

$$
z \longrightarrow \sum_{i}\left(\int_{Q\left(z_{i}, \gamma \theta \delta\left(z_{i}\right)\right)}|F(\zeta)|^{p} \mu(\zeta)^{\beta}(\zeta) d V_{\alpha}(\zeta)\right)^{1 / p} L_{i}(z),
$$


on obtient que

$$
\|F-G\|_{\alpha, \beta, p} \leq C \eta^{1 / m}\|F\|_{\alpha, \beta, p}
$$

car les $Q\left(z_{i}, \gamma \theta \delta\left(z_{i}\right)\right)$, qui sont presques disjoints, entraînent que

$$
\sum_{i} \int_{Q\left(z_{i}, \gamma \theta \delta\left(z_{i}\right)\right)}|f(\zeta)|^{p} \mu(\zeta)^{\beta} d V_{\alpha}(z) \leq C\|F\|_{\alpha, \beta, p}^{p} .
$$

D'après la Proposition 5.4, il existe $C>0$ tel que $\|F-G\|_{\alpha, \beta, p} \leq$ $C \eta^{1 / m}\|F\|_{\alpha, \beta, p}$. Il suffit alors de choisir $0<\eta \leq(1 / 2 C)^{m}$ pour obtenir

$$
\|F-G\|_{\alpha, \beta, p} \leq \frac{1}{2}\|F\|_{\alpha, \beta, p} .
$$

Pour conclure la démonstration du Théorème $\mathrm{D}$, on fixe $0<\eta<\eta_{0}$, $\left(w_{i}\right)$ un $\eta$-réseau sur $\Omega$. Soient $F$ dans $A^{p}\left(\mu(\zeta)^{\beta} d V_{\alpha}\right)$ et $G_{0}$ la fonction discrétisée associée et donnée par la relation 5.6. Pour tout entier $N \geq 1$, on note $G_{N}$ la fonction discrétisée associée à $F-\sum_{0}^{N-1} G_{i}$. On obtient alors,

$$
\left\|F-\sum_{i=0}^{N} G_{i}\right\|_{\alpha, \beta, p} \leq 2^{-N}\|F\|_{\alpha, \beta, p} .
$$

Il suffit alors de noter que $F=\sum_{0}^{\infty} G_{i}$ pour achever la démonstration du Théorème D.

Dans la démonstration précédente, lorsque $p, \alpha$ et $\beta$ sont fixés, les fonctions $K_{i}$ sont définis à partir de $H_{n}(z, \zeta)$ póur $n$ suffisamment grand, ce qui permet de montrer le théorème de décomposition atomique pour $n>n_{2}$. On part de ce résultat pour affaiblir la condition sur $n$ et étendre la Proposition $\mathrm{C}$ et le Théorème $\mathrm{D}$ à $n_{0}=n_{1}$. On commence par supposer $1<p<+\infty$. Dans ce cas, lorsque l'on considère $F$ dans $A^{p}\left(\mu(\zeta)^{\beta} d V_{\alpha}\right)$, il existe $\left(\lambda_{i}\right)$ dans $\ell^{p}$ telle que $F(z)=\sum_{i} \lambda_{i} K_{i}(z)$ et les fonctions $K_{i}(z)$ sont définies à partir du noyau $H_{n}(z, \zeta)$ pour $n>n_{2}$. Pour tout $N \in \mathbb{N}$, on pose $F_{N}(z)=\sum_{i=0}^{N} \lambda_{i} K_{i}(z)$. Le Corollaire 4.2 entraîne $H_{n} F_{N}=F_{N}$. L'opérateur $H_{n}$ étant continu pour $n>n_{1}$, on obtient alors la Proposition $\mathrm{C}$ pour $n_{0}=n_{1}$. On en déduit alors le Théorème $\mathrm{D}$ pour $n_{0}=n_{1}$ lorsque $1<p<+\infty$.

On suppose maintenant $0<p \leq 1$, pour établir la Proposition $\mathrm{C}$, il suffit de montrer l'inclusion suivante, dont la démonstration est identique à celle du Lemme 4.2:

Lemme 5.5. Soient $0<p \leq 1, \alpha$, $\beta$, tels que $1+\alpha+\frac{\beta}{h(\beta)}>0$ et $n$ dans $\mathbb{N}$ tel que $n>n_{1}(\alpha, \beta, p)=\frac{1}{p}\left(\alpha+\frac{\beta}{h(-\beta)}\right)+2\left(1-\frac{1}{p}\right)\left(1+\frac{1}{h(-\beta)}\right)$. Il existe $r>1$ et $-1<a<r n+r-1$ tels que

$$
A^{p}\left(\mu(\zeta)^{\beta} d V_{\alpha}\right) \subseteq A^{r}\left(d V_{a}\right)
$$


On obtient alors la Proposition C pour $n_{0}=n_{1}$, ce qui permet encore de prouver le Théorème $\mathrm{D}$ pour $n_{0}=n_{1}$.

\title{
References
}

1. D. Catlin, Estimates of invariant metrics on pseudoconvex domains of dimension two, Math. Zeit. 200 (1989), 429-466.

2. R. Coifman et G. Weiss, "Analyse harmonique non commutative sur Certains Espaces Homogènes," Lecture Notes in Mathematics 242, Springer-Verlag, 1971.

3. R. Coifman et R. Rochberg, Representation theorem for holomorphic and harmonic functions in $L^{p}$, S.M.F., Asterisque $\mathbf{7 7}$ (1980), 1-65.

4. B. Coupet, Décomposition atomique des espaces de Bergman, Indiana Math. J. 38 (1989), 917-941.

5. K. Diederich et J. E. Fornaess, Pseudoconvex domains: bounded strictly plurisubharmonic exhaustion function, Invent. Math. 39 (1977), 129-141.

6. F. Forelli et W. Rudin, Projections on spaces of holomorphic functions in balls, Indiana Univ. J. 24(6) (1974), 593-602.

7. E. LigockA, On the Forelli-Rudin construction and weighted Bergman projection, Studia Math. 94 (1989), 257-272.

8. J. MAC-NEAL, Boundary behaviour of the Bergman kernel function in $\mathbb{C}^{2}$, Duke Math. 58 (1989), 499-512.

9. A. Nagel, J.-P. Rosay, E. Stein et S. Wainger, Estimates for the Bergman and the Szegö kernel in $\mathbb{C}^{2}$, Ann. of Math. 129 (1989), $113-149$.

10. K. ZHU, "Operator Theory in function spaces," Decker, New York, 1990.

1991 Mathematics subject classifications: 32F15, 32H10

\author{
Département de Mathématiques \\ Université de Poitiers \\ 40, Avenue du Recteur Pineau \\ 86022 Poitiers \\ FRANCE
}

Primera versió rebuda el 28 de Novembre de 1994, darrera versió rebuda el 5 de Juliol de 1995 